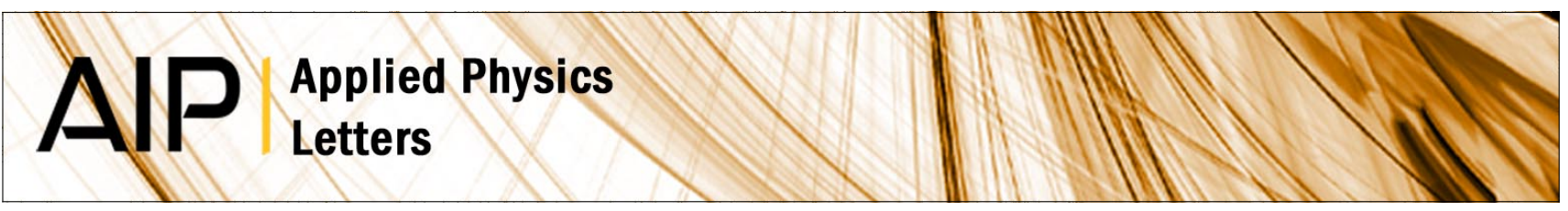

\title{
Ultrafast gain dynamics in InP quantum-dot optical amplifiers
}

Wolfgang Langbein, Valentina Cesari, Francesco Masia, Andrey B. Krysa, Paola Borri et al.

Citation: Appl. Phys. Lett. 97, 211103 (2010); doi: 10.1063/1.3518715

View online: http://dx.doi.org/10.1063/1.3518715

View Table of Contents: http://apl.aip.org/resource/1/APPLAB/v97/i21

Published by the American Institute of Physics.

\section{Related Articles}

Electroluminescence from strained germanium membranes and implications for an efficient Si-compatible laser Appl. Phys. Lett. 100, 131112 (2012)

A weakly coupled semiconductor superlattice as a potential for a radio frequency modulated terahertz light emitter

Appl. Phys. Lett. 100, 131104 (2012)

Quantum-dot nano-cavity lasers with Purcell-enhanced stimulated emission

Appl. Phys. Lett. 100, 131107 (2012)

Effect of internal optical loss on the modulation bandwidth of a quantum dot laser

Appl. Phys. Lett. 100, 131106 (2012)

Design of three-well indirect pumping terahertz quantum cascade lasers for high optical gain based on nonequilibrium Green's function analysis

Appl. Phys. Lett. 100, 122110 (2012)

\section{Additional information on Appl. Phys. Lett.}

Journal Homepage: http://apl.aip.org/

Journal Information: http://apl.aip.org/about/about_the_journal

Top downloads: http://apl.aip.org/features/most_downloaded

Information for Authors: http://apl.aip.org/authors

\section{ADVERTISEMENT}

\section{ACCELERATE AMBER AND NAMD BY $5 X$.}

NVIDIA TRYIT ONA FREE, REMOTELYYHOSTED CLUSTER. 


\title{
Ultrafast gain dynamics in InP quantum-dot optical amplifiers
}

\author{
Wolfgang Langbein, ${ }^{1, a)}$ Valentina Cesari, ${ }^{1}$ Francesco Masia, ${ }^{1}$ Andrey B. Krysa, ${ }^{2}$ \\ Paola Borri, ${ }^{1,3}$ and Peter M. Smowton ${ }^{1}$ \\ ${ }^{1}$ School of Physics and Astronomy, Cardiff University, The Parade, Cardiff CF24 3AA, United Kingdom \\ ${ }^{2}$ EPSRC National Centre for III-V Technologies, University of Sheffield, Sheffield S1 3JD, United \\ Kingdom \\ ${ }^{3}$ School of Biosciences, Cardiff University, Museum Avenue, Cardiff CF10 3AX, United Kingdom
}

(Received 16 October 2010; accepted 2 November 2010; published online 22 November 2010)

\begin{abstract}
We measured the gain dynamics at the ground-state transition in an electrically pumped InP/ AlGaInP quantum-dot optical amplifier at room temperature by femtosecond differential transmission. The gain shows an ultrafast recovery within $200 \mathrm{fs}$, even faster than in state-of-the-art InAs/GaAs quantum-dot amplifiers. This finding, likely to be due to the less confined and more closely spaced hole levels in InP dots, is promising for optical signal processing at high bit rates. We furthermore measured the pump-induced refractive index changes and deduced a linewidth enhancement factor similar to the one in InAs/GaAs quantum dots. (c) 2010 American Institute of Physics. [doi:10.1063/1.3518715]
\end{abstract}

The application of semiconductor quantum dots (QDs) in optoelectronics has been a major research theme during the past 15 years, driven by predictions of superior performance in these zero-dimensional systems compared to bulk and quantum-well based devices. Recently, epitaxially grown InP/AlGaInP QDs have been investigated for application in diode lasers covering the $650-780 \mathrm{~nm}$ wavelength range, ${ }^{1,2}$ important for dual wavelength data storage, plastic fiber communications, and bioimaging. InP/GaInP QDs also provide single-photon sources for quantum cryptography protocols at wavelengths better matched to silicon photodetectors. $^{3}$

Compared to the widely studied InAs/GaAs QD lasers and amplifiers, the optoelectronic properties of InP/GaInP QD devices are rather unexplored. Gain characteristics have been determined in a few reports, ${ }^{4,5}$ but their gain recovery dynamics are still unknown. On the other hand, gain dynamics are crucial not only to determine the intrinsic speed limit for signal processing applications at high bit rates but also as a probe of fundamental physical processes such as carriercarrier and carrier-phonon scattering.

In this work, we have measured the ultrafast gain recovery dynamics of the ground-state transition in electrically pumped InP/AlGaInP QD optical amplifiers at room temperature. The structures were grown on n-GaAs (100) substrates, oriented $10^{\circ}$ off toward [111], by metal organic vapor phase epitaxy in a low-pressure, horizontal flow reactor, using trimethyl precursors for the group III elements and arsine $\mathrm{AsH}_{3}$ and phosphine $\mathrm{PH}_{3}$ as precursors of the group $\mathrm{V}$ elements. The structure investigated here was grown at a temperature of $730{ }^{\circ} \mathrm{C}$ and its core consisted of five QD layers, each formed by $2.5 \mathrm{ML}$ of InP grown on $\left(\mathrm{Al}_{0.3} \mathrm{Ga}_{0.7}\right)_{0.51} \mathrm{In}_{0.49} \mathrm{P}$, covered by a $8 \mathrm{~nm} \mathrm{Ga} \mathrm{G}_{0.51} \mathrm{In}_{0.49} \mathrm{P}$ quantum well (QW) and separated by $16 \mathrm{~nm}$ wide $\left(\mathrm{Al}_{0.3} \mathrm{Ga}_{0.7}\right)_{0.51} \mathrm{In}_{0.49} \mathrm{P}$ barrier layers. This core was clad on both sides with $1 \mu \mathrm{m}$ of $\mathrm{Al}_{0.51} \mathrm{In}_{0.49} \mathrm{P}$ (see sketch in Fig. 1), $\mathrm{n}$-doped at $1 \times 10^{18} / \mathrm{cm}^{3}$, and p-doped at $3 \times 10^{17} / \mathrm{cm}^{3}$, respectively. All layers except InP are nominally lattice

${ }^{\text {a)} E l e c t r o n i c ~ m a i l: ~ l a n g b e i n w w @ c a r d i f f . a c . u k . ~}$ matched to GaAs. The structure was patterned with a $50 \mu \mathrm{m}$ wide oxide isolated stripe and cleaved along the $[01 \overline{1}]$ facet orthogonal to the stripe to define a $300 \mu \mathrm{m}$ long waveguide. Both optical facets were coated with a $\lambda / 4$ layer of $\mathrm{HfO}_{2}$ to

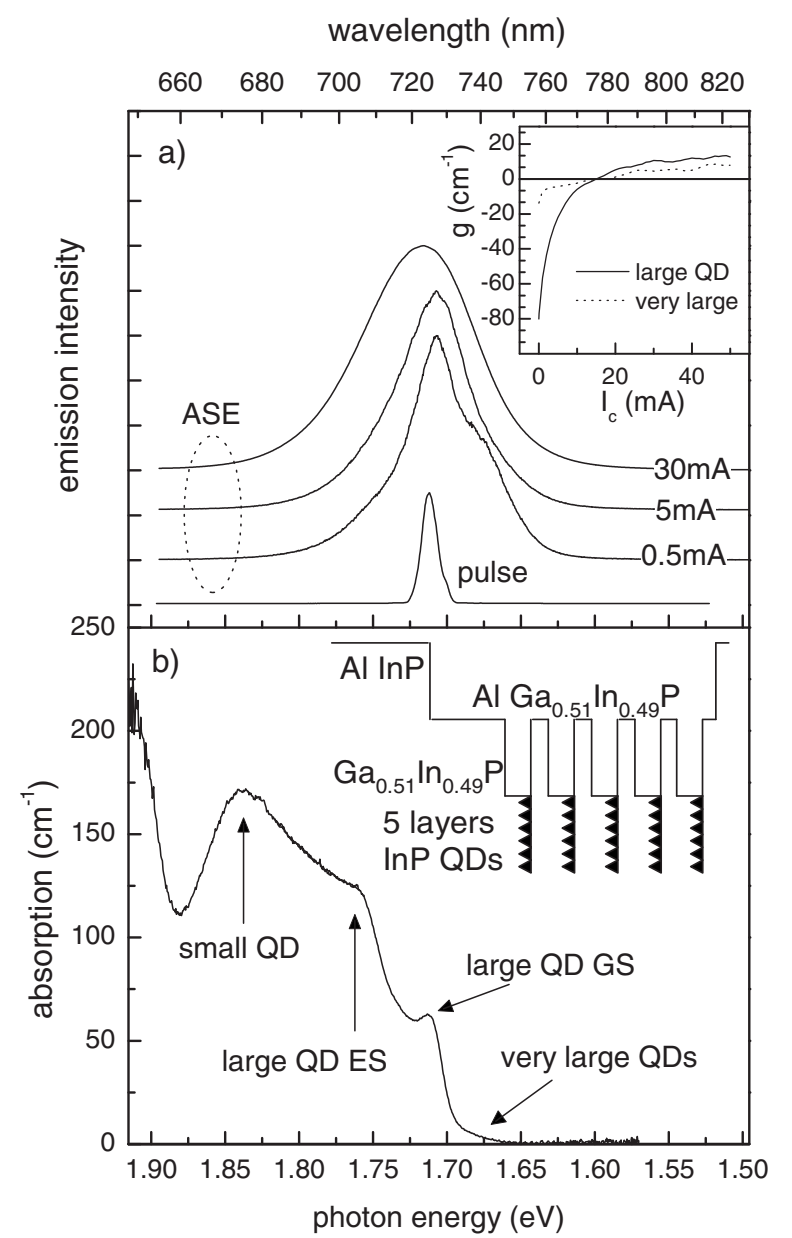

FIG. 1. Spectra of (a) amplified spontaneous emission and (b) absorption from the investigated InP QD optical amplifier. A sketch of the sample structure is given. The top inset shows the modal gain at wavelengths of 720 (large QDs) and $740 \mathrm{~nm}$ (very large QDs). The spectrum of the pulses used in the pump-probe experiment is shown in (a). 
allow for single pass transmission experiments by suppressing back reflections.

Atomic force microscopy of QDs grown under similar conditions showed a bimodal distribution of QD sizes, ${ }^{4}$ not unusual in these systems. ${ }^{6}$ The absorption spectrum measured at room temperature on a structure grown under nominally identical conditions [see Fig. 1(b)] exhibited three resonances at $\sim 1.71,1.76$, and $1.84 \mathrm{eV}$, which we attribute to the ground-state (GS) and excited-state (ES) transitions of the larger QDs and the GS transition of the smaller QDs, respectively. This attribution is motivated by absorption measurements on a set of samples where the features attributed to the large QDs grow proportional to the quantity of deposited InP, whereas those attributed to the small QDs shrink. The onset of the two-dimensional continuum from the GaInP QW absorption is visible at $\sim 1.91 \mathrm{eV}$, determining the confinement energy of the large dots (i.e., the energetic distance of GS and QW transition) to about $200 \mathrm{meV}$. The amplified spontaneous emission (ASE) spectra [Fig. 1(a)] of the investigated QD amplifier at very low injection current show a further resonance around $1.67 \mathrm{eV}$ attributed to a tail of very large dots in the size distribution. A corresponding tail is visible also in the absorption spectrum. With increasing injection current, the ASE spectrum becomes dominated by the GS and ES emission from the large dots. The attribution of the tail to very large dots is supported by measurements of the modal gain using the transmission of a weak optical probe resonant to the GS of each dot subset [see inset of Fig. 1(a)]. The inferred dependencies of the modal gain on injection current show similar transparency currents for both transitions, which is not consistent with an attribution of the two resonances to GS and ES of the same dots, as the ES is expected to have a larger transparency current due to intradot thermal equilibrium. For transitions of different QDs, on the other hand, mutual thermal equilibrium might not be reached and the observed behavior is reconcilable.

Gain and refractive index dynamics were measured using a pump-probe differential transmission technique in heterodyne detection, ${ }^{7-9}$ using $\sim 100$ fs Fourier-limited optical pulses at $76 \mathrm{MHz}$ repetition rate in resonance with the GS of the large dots [see Fig. 1(a)]. The optical excitation was coupled into and out of the device using 0.65 numerical aperture lenses. To account for the strong vertical optical confinement in absence of lateral confinement, a pair of cylindrical lenses was used to create an elliptical optical mode of about $1 \mu \mathrm{m}$ height and $5 \mu \mathrm{m}$ width (intensity full width at half maximum) at the input facet. The width was chosen such that the resulting Rayleigh length of about $120 \mu \mathrm{m}$ in the waveguide was similar to half the waveguide length and by placing the tail of the mode in the center of the waveguide, we ensure an approximately constant width within waveguide, limiting the related pump-intensity variation.

The pump-induced change of the gain $\Delta g$ deduced from the probe transmission change is shown in Figs. 2(a) and 2(b) versus pump-probe delay time $\tau_{\mathrm{P}}$ (positive when the pump pulse is leading) for different injection currents corresponding to absorption and gain regimes. Measurements were taken using a constant input pump intensity. Fits of the dynamics (solid lines) are obtained by convoluting the pulse intensity autocorrelation with a fourfold exponential response function and an instantaneous contribution accounting for two-photon absorption and coherent artifact. ${ }^{10}$ For comparison, gain dynamics of a $1.3 \mu \mathrm{m} \mathrm{InAs/GaAs} \mathrm{QD} \mathrm{op-}$

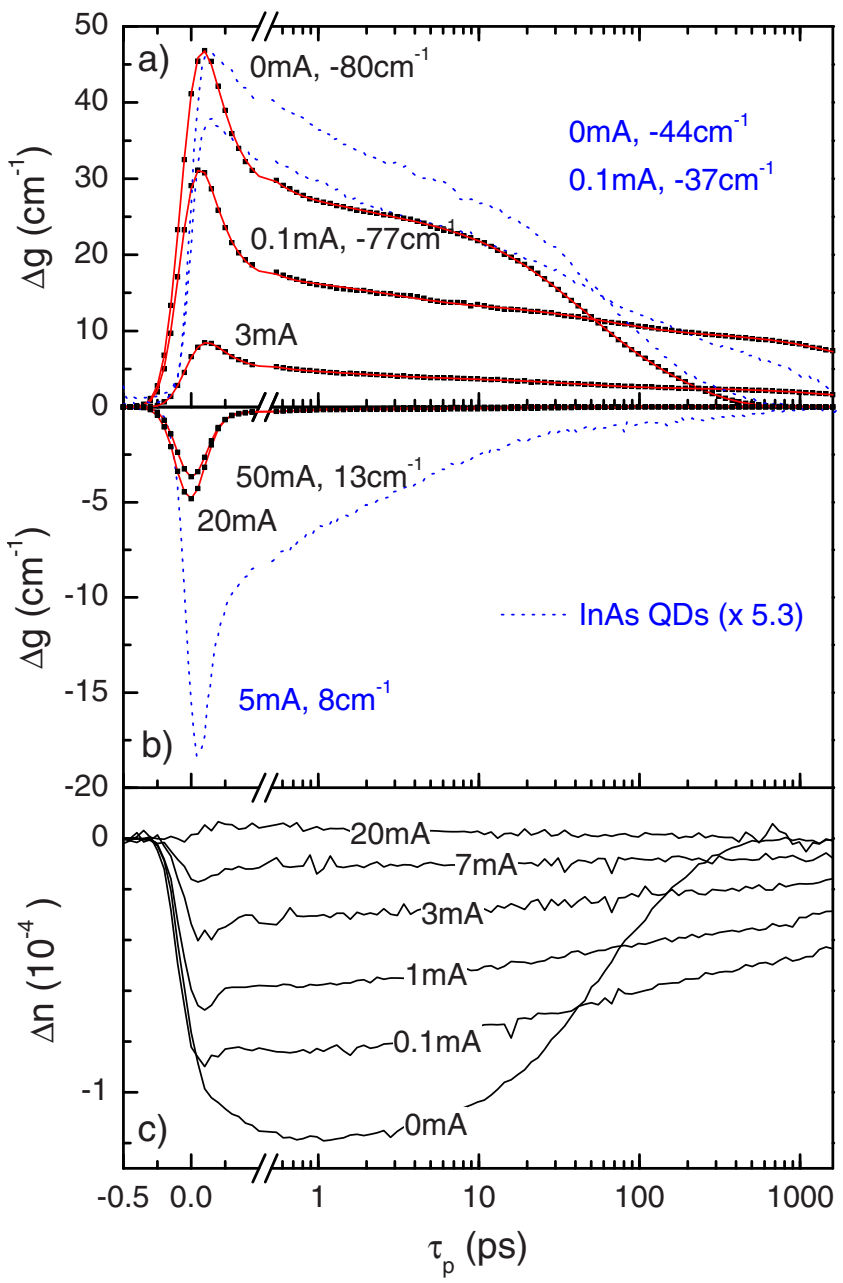

FIG. 2. (Color online) [(a) and (b)] Pump-induced gain (symbols) and (c) refractive index dynamics of the InP QD optical amplifier. The gain dynamics of an InAs/GaAs QD amplifier (Refs. 7 and 10) are shown for comparison (dashed curves). Injection currents and corresponding modal gains are indicated.

tical amplifier (dashed lines) are also shown, ${ }^{7,10}$ scaled by a common factor of 5.3 to match the absorption bleaching at zero injection current.

In the absence of electrical injection $(0 \mathrm{~mA})$, the QDs are initially empty and the pump-induced absorption bleaching (positive $\Delta g$ ) recovers on the time scale on which carriers, generated in the GS by the pump, thermalize into the higher energy states within and outside the QDs, followed by the overall density lifetime via recombination processes. In InAs/GaAs QDs, the initial intradot thermalization occurs on a few picoseconds time scale. Conversely, in the InP QDs, the initial recovery of the absorption bleaching is ultrafast, dominated by a subpicosecond component (from the fit to the data, we find that the dynamics with time constants $<0.2$ ps contribute to $64 \%$ of the total response amplitude). In the absence of injection current, we would exclude that carriercarrier scattering contribute to the dynamics, unless dots are unintentionally doped which appears unlikely given the large value of measured absorption in these conditions $\left(-80 \mathrm{~cm}^{-1}\right)$. We thus attribute the ultrafast recovery of the absorption bleaching to rapid carrier-phonon scattering among closely spaced energy levels in InP dots. Compared to InAs/GaAs QDs with typical dot sizes of $\sim 20 \mathrm{~nm}$ width and $\sim 5 \mathrm{~nm}$ height, InP/GaInP QDs are larger, having $\sim 40 \mathrm{~nm}$ 
typical widths and $\sim 5 \mathrm{~nm}$ height for the small QDs and $\sim 15 \mathrm{~nm}$ height for the large dots. ${ }^{3,6,11}$ Calculations indeed report closely spaced (few meV) hole energy levels in InP QDs since the holes are heavier and also more weakly confined via a shallow valence band potential entirely due to strain, with unstrained InP having a valence band alignment of type II. ${ }^{11}$

With increasing injection current, the dynamics becomes dominated by an ultrafast recovery below the duration of pump and probe pulses manifesting as a reduced $\Delta g$ experienced by the probe and a temporal dynamics tending to the pulse intensity autocorrelation. This is attributed to intradot carrier-carrier scattering. Surprisingly, this effect is already prominent at a low injection current of $0.1 \mathrm{~mA}$, where the injected density results in only a $4 \%$ reduction of the absorption, showing that the average occupation of the involved states is $2 \%$. By fitting the data at $0.1 \mathrm{~mA}$, we find that the dynamics with time constants $<0.2$ ps contribute to $87 \%$ of the total amplitude. The speeding up of the dynamics from 0 to $0.1 \mathrm{~mA}$ is confirmed in the pump-induced change of the refractive index [Fig. 2(c)], where the buildup of a negative $\Delta n$ reflects the thermalization of the optically generated carriers into higher energy states. The finding that already a small state occupation by electrically injected carriers significantly accelerates the gain recovery dynamics is surprising, and not observed in the InAs/GaAs QDs [see dashed lines at 0 and $0.1 \mathrm{~mA}$ in Fig. 2(a)]. This behavior of the InP QDs could be due to the weak confinement and large mass of the holes, resulting in a dominant occupation of ES and wetting layer and fast hole scattering into the continuum. The long overall density lifetime, $5 \mathrm{~ns}$ at $0.1 \mathrm{~mA}$, indicates weak nonradiative recombination and thus a good sample quality. At $0 \mathrm{~mA}$, the density lifetime is significantly shorter due to carrier escape to the contacts provoked by the built-in electric field at zero bias voltage across the $\mathrm{p}$-i-n diode. The quantum-confined stark effect arising from this electric field influences the level structure in these rather large QDs, providing an additional mechanism which could be underlying the observed changes in the dynamics between 0 and 0.1 $\mathrm{mA}$.

In the gain regime, the GS is occupied with injected carriers and the pump induces a gain reduction via stimulated emission (negative $\Delta g$ ), which recovers via carrier relaxation from the higher energy states into the GS. We observe an extremely fast gain recovery below our time resolution. Fitting the dynamics shows that time constants $<0.1$ ps contribute to $98 \%(96 \%)$ of the total amplitude at $50 \mathrm{~mA}(20$ $\mathrm{mA})$. Remarkably, for InAs/GaAs QDs at an injection current yielding an equal modal gain relative to the maximum absorption (and at an injection current roughly twice the transparency current in both systems), the gain dynamics is much slower, with a substantial recovery on the few picoseconds time scale. This result is again likely to be due to the less confined and more closely spaced hole levels and consequent faster carrier relaxation into the GS from the ES and continuum states in InP QDs compared to InAs/GaAs QDs.

From the measured pump-induced gain and refractive index changes at various injection currents, we have inferred the linewidth enhancement factor (LEF), also called

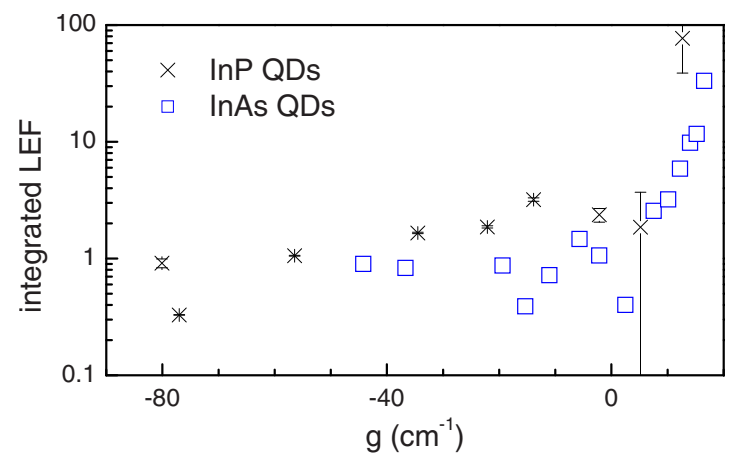

FIG. 3. (Color online) Linewidth enhancement factor for a thermalized carrier distribution (see text) vs small signal gain in the InP/AlGaInP QD optical amplifier (cross) compared to the InAs/GaAs amplifier (open square).

$\alpha$-parameter, in the same way as reported for the InAs/GaAs QD optical amplifier. ${ }^{9}$ In particular, to infer a value of LEF solely due to thermalized carrier-density changes, we have time integrated the pump-induced gain and refractive index change for $\tau_{\mathrm{P}}>100 \mathrm{ps}$. The results are given in Fig. 3 versus modal gain $g$ and are compared with the LEF measured in the InAs/GaAs QD amplifier. Due to the ultrafast recovery dynamics in the gain regime, there is little pump-induced gain and phase change present after $100 \mathrm{ps}$, leading to a large uncertainty in the deduced LEF as indicated by the error bars. The LEF is about 1 at very low injection currents and increases with increasing current to about 2, slightly larger than for the InAs QDs.

In conclusion, the absorption and gain recovery dynamics in InP/AlGaInP QDs are dominated by a sub-200 fs component, eventually contributing to $98 \%$ of the total response in the gain regime. This response is much faster than in previously investigated InAs/GaAs quantum-dot amplifiers and is promising for applications of InP QDs as fast saturable absorbers and amplifiers for signal processing at $>100 \mathrm{GHz}$ rates.

This work was supported by the UK Engineering and Physical Sciences Research Council (EPSRC) Grant No. EP/ E056385. The sample was processed by Karen Barnett.

${ }^{1}$ G. Walter, J. Elkow, N. Holonyak, Jr., R. D. Heller, X. B. Zhang, and R. D. Dupuis, Appl. Phys. Lett. 84, 666 (2004).

${ }^{2}$ J. Lutti, P. Smowton, G. Lewis, A. Krysa, J. Roberts, P. Houston, Y. Xin, Y. Li, and L. Lester, Electron. Lett. 41, 247 (2005).

${ }^{3}$ W.-M. Schulz, R. Rossbach, M. Reischle, G. J. Beirne, M. Bommer, M. Jetter, and P. Michler, Phys. Rev. B 79, 035329 (2009).

${ }^{4}$ J. Lutti, P. M. Smowton, G. M. Lewis, P. Blood, A. B. Krysa, J. C. Lin, P. A. Houston, A. J. Ramsay, and D. J. Mowbray, Appl. Phys. Lett. 86, $011111(2005)$.

${ }^{5}$ P. M. Smowton, M. S. Al-Ghamdi, S. Shutts, G. Edwards, M. Hutchings, and A. B. Krysa, IEEE Photonics Technol. Lett. 22, 88 (2010).

${ }^{6}$ J. Persson, M. Holm, C. Pryor, D. Hessman, W. Seifert, L. Samuelson, and M.-E. Pistol, Phys. Rev. B 67, 035320 (2003).

${ }^{7}$ V. Cesari, W. Langbein, P. Borri, M. Rossetti, A. Fiore, S. Mikhrin, I. Krestnikov, and A. Kovsh, Appl. Phys. Lett. 90, 201103 (2007).

${ }^{8}$ P. Borri, S. Schneider, W. Langbein, and D. Bimberg, J. Opt. A, Pure Appl. Opt. 8, S33 (2006).

${ }^{9}$ V. Cesari, P. Borri, M. Rossetti, A. Fiore, and W. Langbein, IEEE J. Quantum Electron. 45, 579 (2009).

${ }^{10}$ V. Cesari, W. Langbein, P. Borri, M. Rossetti, A. Fiore, S. Mikhrin, I. Krestnikov, and A. Kovsh, IET Optoelectron. 1, 298 (2007).

${ }^{11}$ C. Pryor, M.-E. Pistol, and L. Samuelson, Phys. Rev. B 56, 10404 (1997). 